\title{
Pulse shape effects on the measurement of Temperature using a Brillouin based Optical fiber sensor
}

\author{
Carlos Galindez ${ }^{*}$, Francisco-Javier Madruga, Adolfo Cobo, Olga Conde, Jose-Miguel Lopez- \\ Higuera \\ Photonics Engineering Group, Department TEISA, Universidad de Cantabria \\ ETSII y de Teleco, Avda Los Castros s/n, 39005 Santander-Spain
}

\begin{abstract}
Distributed fiber sensing based on Brillouin gain scattering (BGS) principle is a useful way to develop devices capable to measure temperature or/and strain in optical fibers. New effects or technologies that could achieve a larger distance and/or a better spatial resolution are a topic of special interest in this fiber sensing area. The influence of the probe-pulse shape in the interaction between the pulsed light and the continuous wave laser in a pump-probe system is presented. The purpose of this study is to improve the spatial resolution of the measurement without losing stability in the BGS. Also it is showed how the backscattering Brillouin gain is affected by inducing variations on the final value of the BGS intensity; this effect is illustrated by using an experimental set up based on the Brillouin optical time-domain analysis (BOTDA). Theoretical analysis of the probe pulse in the Brillouin shift and intensity value using triangular, sinc and saw tooth shapes around the medium phonon life time $(\sim 10 \mathrm{~ns})$ are presented; as well as the experimental results and possible applications are explained.
\end{abstract}

Keywords: Brillouin gain scattering, distributed temperature measurements, Optical time domain analysis, probe pulse shape.

\section{INTRODUCTION}

Since the Brillouin scattering was for the first time proposed for sensing [1] many works have been presented to realize temperature or strain sensing using fiber optics technology [2]. An appropriate continuous pump light, through the electrostrictive mechanism, can cause fluctuations in the core of an optical fiber, which induces the non linear Brillouin backscattering effect [3]. By using a shift light as a probe signal, this effect is successfully employed to develop distributed sensing of temperature and/or strain along a fiber, since the Brillouin frequency $v_{\mathrm{B}}$ is function of these two parameters. Both spontaneous or Stimulated Brillouin scattering can be used in the construction of distributed sensors [4].

The pump and probe lights are generated from the same light source, this method avoids the usage of tunable lasers and problems of stability; the signals are obtained by splitting the source light via a $3 \mathrm{~dB}$ coupler, one of its branch is modulated using an electro-optic intensity modulator, when the modulation frequency matches the Brillouin frequency, $v$ $=v_{\mathrm{B}}$, the non linear Brillouin effect reaches its maximum gain, which is determined by monitoring the dependence of the power transfer from one light wave to the other on $v$. In a stimulated Brillouin scattering process a pulsed pump wave and a counter propagating probe wave interact.

In distributed sensors the spatial resolution is one of the most important parameters. In stimulated Brillouin sensors the spatial resolution can be determined by the interaction length between pulse and CW lights, which is half the pulse length. If the CW's frequency is higher than the pulse's frequency, power is transferred from the CW to the pulse. If this light has a lower frequency, the power would transfer from the pulse to the CW light. The first configuration is referred as the loss process since the CW light loses power, the second as the gain process because of the CW power gain $[3,5]$.

Spatial resolution is achieved by using a pulsed and continuous-wave $(\mathrm{CW})$ light source. The spatial resolution is equal to the interaction length between pulse and CW lights, which is half the pulse length.

* carlos.galindez@unican.es; phone \& fax +34 942 200-877; http://gif.teisa.unican.es

Enabling Photonics Technologies for Defense, Security, and Aerospace Applications III, edited by M.J. Hayduk, A.R. Pirich,

P.J. Delfyett Jr., E.J. Donkor, J.P. Barrios, R.J. Bussjager, M.L. Fanto, R.L. Kaminski, G.Li, H.Mohseni, E.W. Taylor, Proc. of SPIE Vol. 6572, 65720S, (2007) -0277-786X/07/\$18 $\cdot$ doi: 10.1117/12.719432

Proc. of SPIE Vol. 6572 65720S-1 
This work studies theoretically the interaction between the pulse and the probe light wave, where the pulse shape is different to the typical square pulse. The interaction with sinc, triangular and saw tooth pulses are studied to analyse the accuracy of Brillouin measurements and the possible shape effects by using more realistic pulses generated in laboratory.

\section{THEORETICAL MODEL}

The description of the SBS process in optical fibers must consider mutual interaction between the pump and stokes waves, for a cw or quasi-cw pump. The steady state coupled-intensity equations for a slowly varying amplitude approximation of the interaction between the scattering and light fields can be expressed as follows $[3,5]$ :

$$
\frac{d I_{i}}{d z}=(\mp) g_{B} I_{i} I_{j} \pm \alpha I_{i} \text { with } i \neq j
$$

where the $\mathrm{i}$ index ( 1 or 2 ) denotes the intensity of the pump or scattering light waves, $\alpha$ is the fiber attenuation coefficient and $g_{B}$ is the Brillouin gain factor. The sign for $g_{B}$ is negative for a Brillouin gain process and positive for a Brillouin loss process. The Brillouin spectral width is related to the damping time of acoustic waves or the phonon lifetime.

In this case the acoustic waves are assumed to decay as $e^{-\frac{t}{T_{B}}}$, with $\mathrm{T}_{\mathrm{B}}$ as the phonon lifetime approximately 10ns for optical fibers. The Brillouin gain scattering (BGS) is described by a Lorentz profile given by

$$
g_{B}(v)=g_{0} \frac{(\Delta v / 2)^{2}}{\left(v-v_{B}\right)^{2}+(\Delta v / 2)^{2}}
$$

where $\Delta v_{\mathrm{B}}$ is the full width at half maximum (FWHM) Brillouin line width and related to the phonon lifetime by $\Delta v_{B}=\left(\pi T_{B}\right)^{-1}$. The Brillouin gain peaks at the Brillouin frequency $v_{\mathrm{B}}$ and is given by [3]

$$
g_{0}=g_{B}\left(v_{B}\right)=\frac{2 \pi n^{7} p_{12}^{1} \gamma}{c \lambda^{2} \rho_{0} V_{a} \Delta v_{B}}
$$

where $p_{12}$ is the longitudinal elasto-optic coefficient, $\rho_{0}$ is the material density, $\lambda$ is the pump light wave, $\mathrm{c}$ is the vacuum velocity of light. The frequency shift $v_{\mathrm{B}}$ in the backward direction is given by $v_{B}=\frac{2 \pi V_{a}}{\lambda}$. The stokes intensity is found to grow exponentially in the backward direction according to

$$
I_{2}(0)=I_{2}(L) e^{g_{0} P o L_{e f f} / A_{e f f}-\alpha L}
$$

this intensity can be express in terms of power by considering $P_{i}=\int I_{i} d A$ in the coupled equations (1), hence the interaction can be write as

$$
P_{i}(z)=P_{i}(0) e^{\int_{L}^{x} a d z} e^{\int_{L}^{x}\left(-g_{B}(v, z)\right) P_{j} / A_{e f f} d z}
$$

where the integration area starts at the fiber length $\mathrm{L}$ and ends at the begin of the pulse wave. 
Hence it is consider the region of the optical fiber where the pulse and pump waves interact; for the system in which both the gain and loss process occur, the total probe light power as a function of distance within the fiber can be expressed as $P_{1}=P_{1}^{+}(z)+P_{1}^{-}(z)$, where the first term on the right-hand side is the power for the gain process and the second term is the power for the loss process and $A_{\text {eff }}$ is the effective fiber core area.

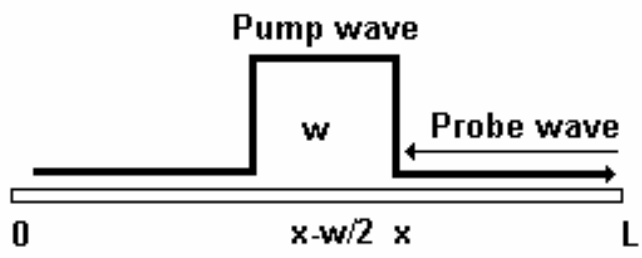

Fig. 1. Interaction between pump and probe light into the optical fiber.

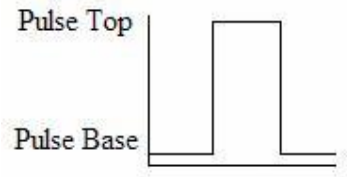

(a)

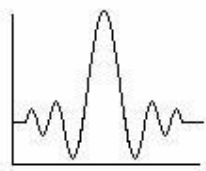

(b)

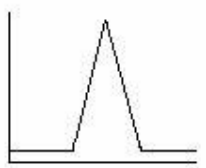

(c)

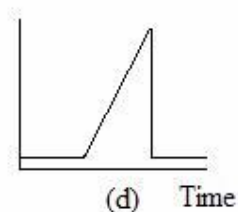

(d) Time

Fig. 2. Pump light shapes that interact with the probe light wave. (a) square pulse, (b) sinc pulse, (c) triangular pulse and (d) saw pulse

In order to use a single laser source, the probe light wave is modulated at a fixed frequency using an external modulator, the modulation gives rise to new frequency lines in the optical spectrum, the so-called modulation sidebands. For pure intensity modulation, the obtained spectrum is symmetrical around the incident light wave frequency and the new lines are equally spaced in frequency. When the modulation frequency is equal to the Brillouin frequency shift $v_{\mathrm{B}}$, the firstorder sidebands can interact with the incident light wave through SBS process, provided propagations in opposite directions. Pulses show a maximum power and a minimum power different to zero, this situation is caused by the non perfect modulation, but if it considered that the pump light has no pulse base or that it is neglected respect to the pulse top, the probe light would interact only with the highest part of the pulse, see Figure 1.

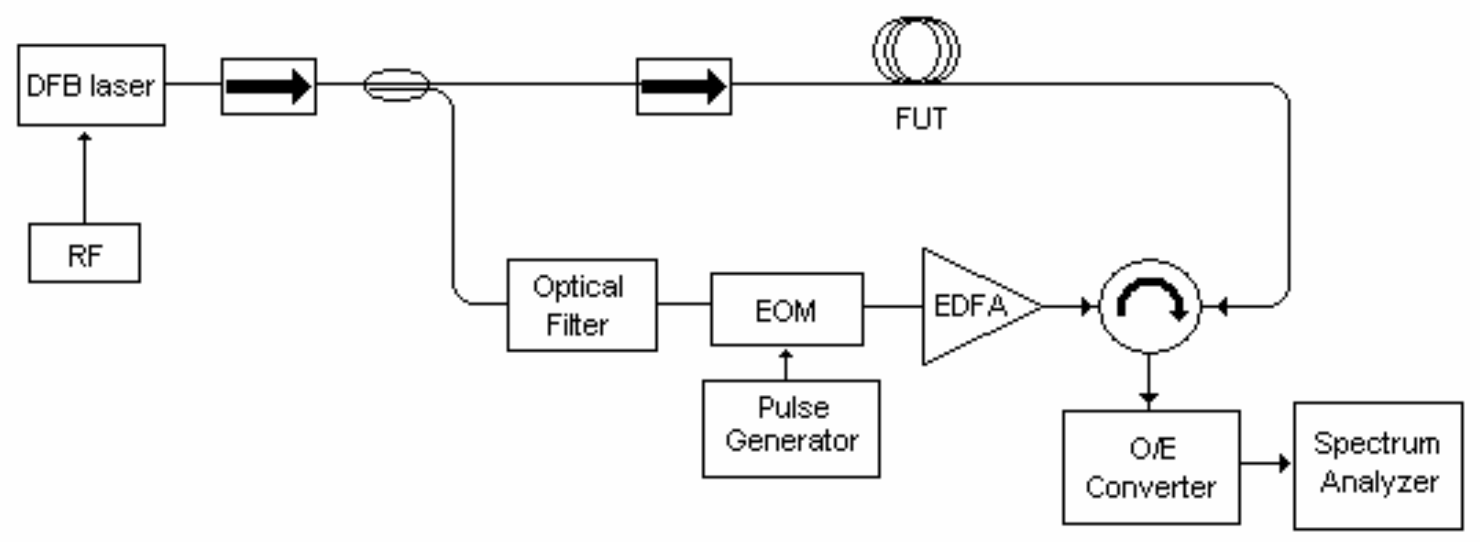

Fig. 3. Experimental setup for Brillouin gain analysis. 


\section{EXPERIMENTAL SETUP}

A DFB laser at $1533.6 \mathrm{~nm}$ with an internal electro-absorption modulator is used as light source, its output is split by a coupler, one branch is amplitude modulated by an electro-optic modulator (EOM) to generate pulses, and then it is amplified by an erbium-doped fiber amplifier (EDFA) and used as the pump light which is launched into the testing fiber. When a single-frequency light wave is modulated at a fixed frequency $f_{\mathrm{m}}$ using an external modulator, the modulation creates new frequency lines in the optical spectrum, the so-called modulation sidebands. For pure intensity modulation, the obtained spectrum is symmetrical around the incident light-wave frequency $V_{0}$, and the new lines are equally spaced in frequency by $f_{\mathrm{m}}$. When the modulation frequency $f_{\mathrm{m}}$ matches the Brillouin frequency $V_{\mathrm{B}}$, the light wave at frequency $v_{0}-f_{\mathrm{m}}$, which are the sidebands, can interact with the incident pump light wave at frequency $v_{0}$ through the Brillouin gain process. In contrast, the light wave at frequency $v_{0}+f_{\mathrm{m}}$, the upper sideband, amplifies the pump light through the Brillouin loss process [1].

The BGS is found by sweeping the modulation frequency. In-line isolators were inserted inside the loop to avoid interference. The BGS was measured by sweeping the modulation frequency $f_{\mathrm{m}}$ in the vicinity of the Brillouin frequency $V_{\mathrm{B}}$ and by detecting the optical power of the probe light using a photo-detector. The output signals of the photo-detector were acquired by a spectrum analyzer. The experimental setup is showed in Fig 3.

\section{SIMULATION AND EXPERIMENTAL RESULTS}

Simulations have been developed by using different pulses, which interact with the backscatter light, those pulses are: square, sinc, triangular, saw tooth and inverted saw tooth, see Fig. 2(a-d). The Brillouin gain factor (BGF) was simulated using equations 1 to 5 , the values used in simulation are: refractive index $n=1.45$, photo-elastic constant $p_{12}=1[5,6]$, density $=2200 \mathrm{~kg} \mathrm{~m}^{-3}$, acoustic wave velocity $V_{\mathrm{a}}=5960 \mathrm{~ms}^{-1}$, polarization factor $\gamma=1$, attenuation coefficient of pump light $\alpha=0.046$, wavelength of the laser $\lambda=1533.6 \mathrm{~nm}$, effective fiber core area $A_{\text {eff }}=50 \mu \mathrm{m}^{2}$.

According to the theory previously described, it is possible to note that the best response in Brillouin gain is obtained with the square pulse, which presents an integration power area (Fig. 1 and 2) greater than the other shapes of similar pulse conditions of pulse top and pulse width power. But the pulse shape that really acts in an experimental set up looks more like a triangular, saw or sinc pulse; where the accuracy of the measurement is influenced both by the total interacting power and the spatial resolution. This situation depends on the pulse generator accuracy and its performance; thus it is convenient to simulate the variation of power and pulse width around phonon lifetime to analyze BGF using these pulse shapes.

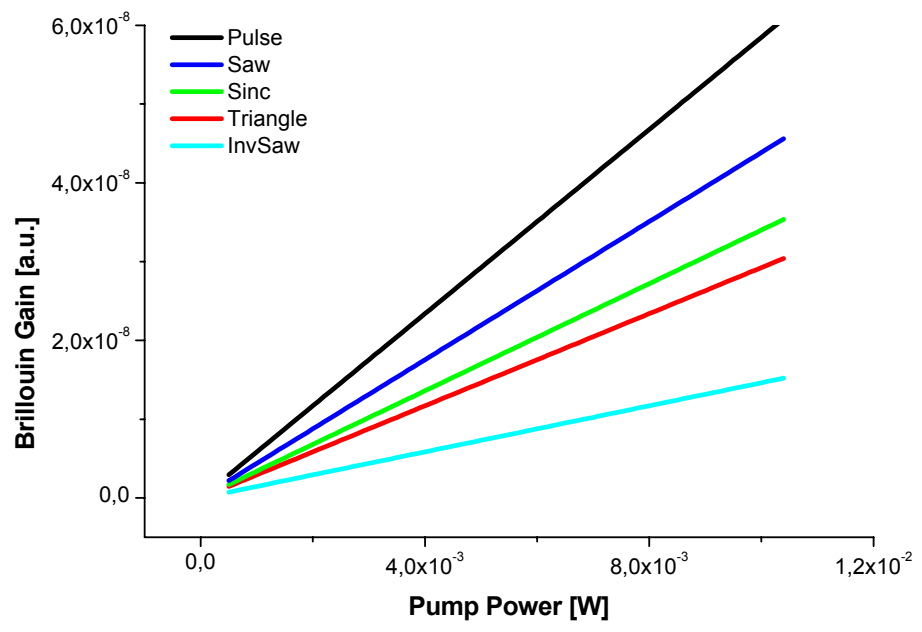




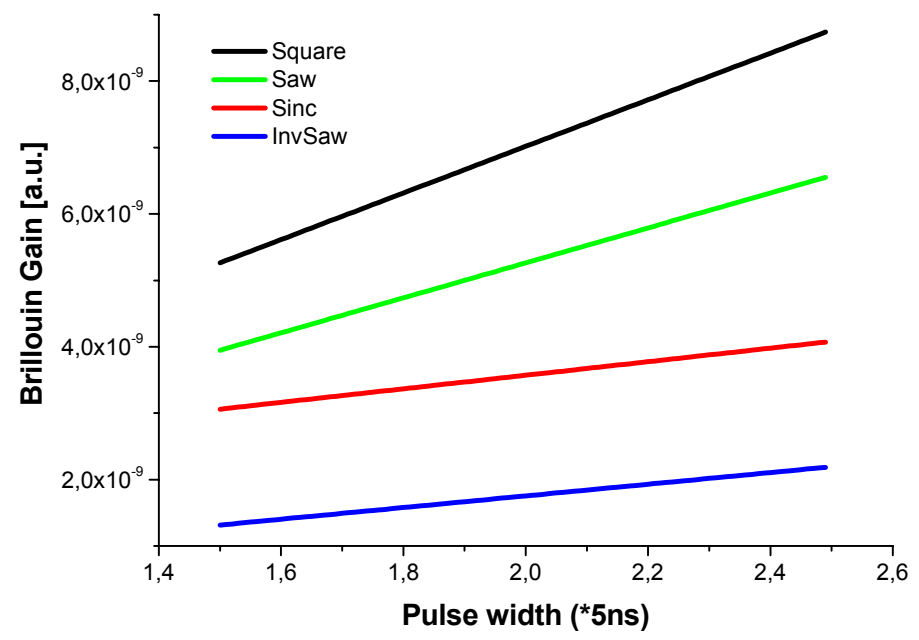

(b)

Fig. 4. Brillouin gain response. (a) Power variation for 10ns pulse width and different pulses, (b) pulse width variation for pulses with power of $1.2 \mathrm{~mW}$.

For a variation of pulse shape BGF presents a better response when the interaction is due to a saw pulse (see Fig 4.a). This gain is enlarged and rapidly achieved when higher amounts of incident power are used. If the study is made for constant pulse width fixed at phonon lifetime of $10 \mathrm{~ns}$ and the power is changed around a constant value, the BGF response is similar to the pulse width analysis which presents an increment that depends on the power and the Brillouin threshold (see Fig 4.b). The best response is presented when the pulse has more power into the interaction area.

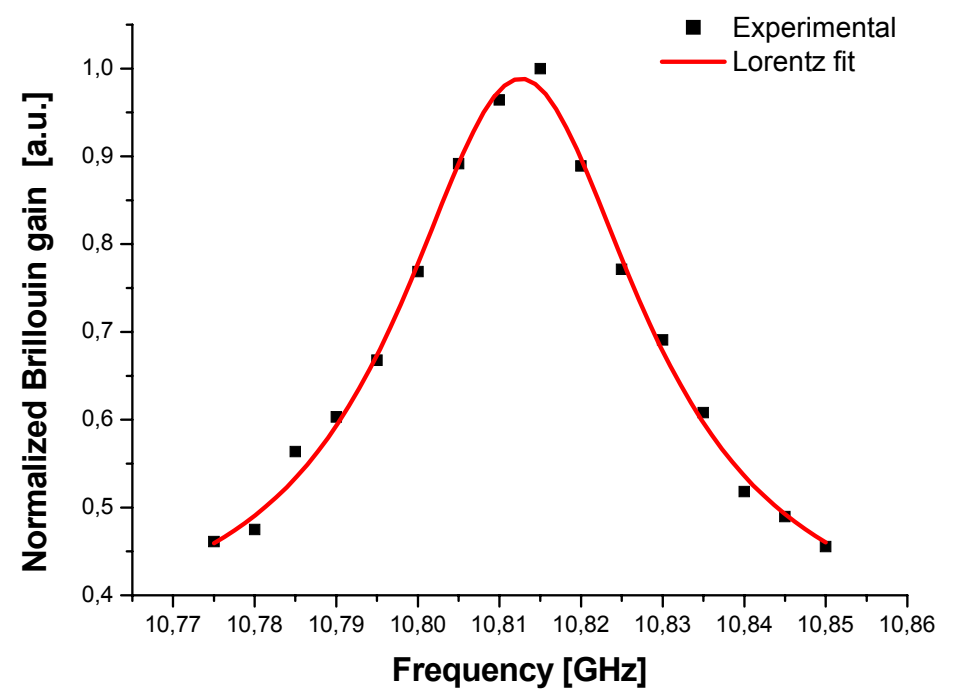

Fig. 5. Brillouin gain spectrum for a $20 \mathrm{~km}$ single mode optical fiber. Experimental data (points) and Lorentz fit (line).

$$
\Delta v_{\mathrm{B}}=32.38 \mathrm{MHz}, v_{\mathrm{B}}=10.81 \mathrm{GHz} \text {. }
$$

Fig. 5 shows experimental results for Brillouin gain spectrum obtain at $25^{\circ} \mathrm{C}$; these values have been used to carry out theoretical approximations showed in fig 4. It is important to note the adequate selection of frequency and power, in order to obtain accuracy in the experimental results. Once the experimental setup and the optical fiber were characterized, it is possible to start with paper's idea, of changing power and pulse width of probe signal. To achieved the experimental purpose, it has to replace the pulse generator by an arbitrary wave form generator, this device is 
programmed to produce saw, triangular and square pulses, when pulses around $10 \mathrm{~ns}$ are analyzed with an oscilloscope, it is observed that the pulse seems to a sinc function more than a perfect square step, of course, this situation depends on the wave generator accuracy.

Consistent with theoretical analysis, for variations of pulse shape, experimental BGF presents a better response when the interaction is due to a saw pulse, see fig. 6; similar results have been obtained theoretically (fig. 4) when the gain is enlarged and rapidly achieved by using higher amounts of incident power. For constant pulse width fixed at phonon lifetime around $10 \mathrm{~ns}$ and changing power above the threshold value, experimental BGF response is similar to theoretical pulse width analysis which presents an increment that depends on the power and the Brillouin threshold, see Fig 7. The best response is presented when the pulse has more power into the interaction area.

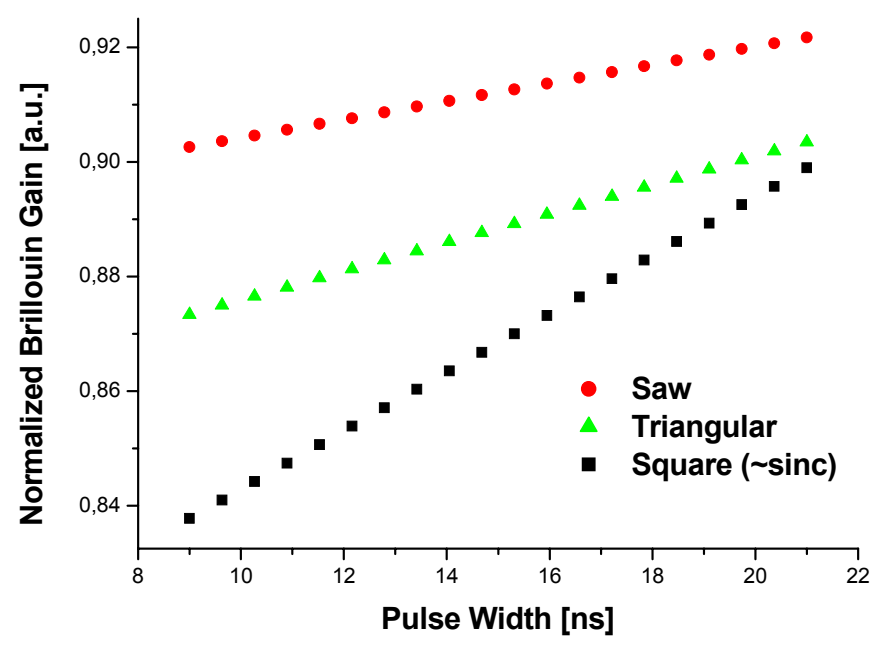

Fig. 6. Experimental Brillouin gain spectrum response for a constant power and a variation of pulse width in saw, triangular and square pulse.

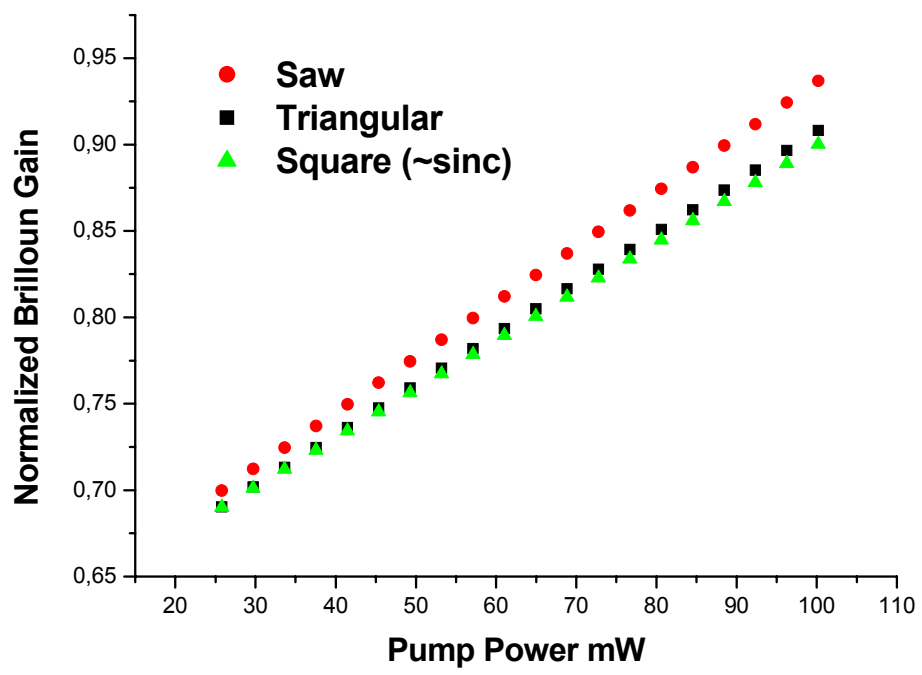

Fig. 7. Experimental Brillouin gain spectrum response when pulse width is around 10ns and the pump power is varied for saw, triangular and square pulses 
Fig 8 presents experimental BGF values when a saw pulse is used at a fix pump power and 3 different widths. The received power depends on the amount of time applied to carry out the interaction between the light and the sound waves which are generated by the high power concentration in the fiber core. Then, in order to obtain accuracy in the measurement of BGF it is important to select a set of power and pulse conditions of saw tooth pulse to obtain similar results to a square pulse, thus this system could allow better approximation and sensitivity of temperature and/or strain measurements.

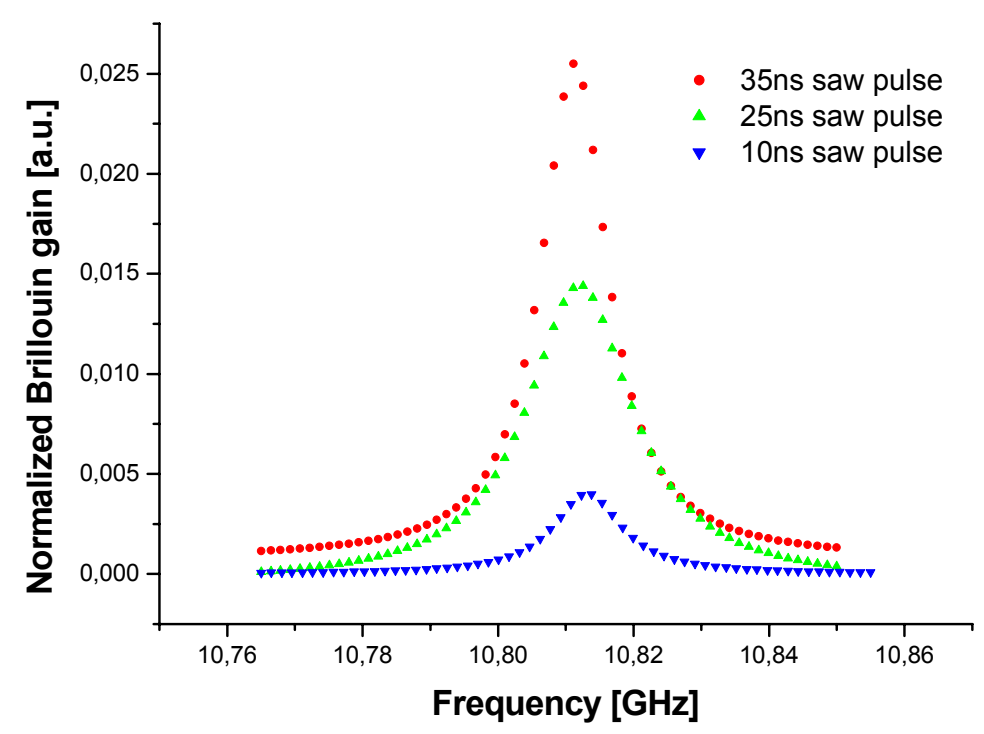

Fig. 8. Experimental Brillouin gain spectrum measured for different saw pulse widths.

\section{CONCLUSIONS}

The behavior of BGF when a pump field and different pulse shape fields interact in an optical fiber system was analyzed and studied theoretically and experimentally in this paper. The results show that the interaction depends on the total amount of power involved during the nonlinear process. Since experimentally pulses are not perfectly squared, it is important to use pulses that present a better approximation, in order to improve the spatial resolution results.

Experimental results fit correctly to simulations and they are consistent with the experimental results reported in fiber sensing papers, which verifies properly the theoretical approach consider in this work. The most important result of this experiment is that it is possible to use other pulse shapes like saw tooth to get similar results in Brillouin gain measurements. It only requires to meet a set of power and pulse conditions of saw tooth pulse close to its square pulse equivalent on power. An improvement on the spatial resolution could be achieved if the power is increased and the pulse width reduced for sinc or saw pulses.

The measurement of temperature by Brillouin back scattering process in an optical fiber, depends on the distance and the step which test every part of the fiber, thus by using this kind of pulse shapes (saw, sinc or triangular pulses) it is possible to obtain better data for the same pulse width than the traditional square pulse under special circumstances.

The importance and the accuracy of this fiber technique make possible its utilization in extreme environmental conditions, additionally it is suitable for controlling temperature or/and strain variables in systems where it is necessary to avoid complexity in sensor connections and accessibility. 


\section{ACKNOWLEDGMENTS}

This work has been supported by the Spanish Government under the research project TEC2004-05936-CO2-02.

\section{REFERENCES}

1. Culverhouse, D., et al., Potential of stimulated Brillouin scattering as sensing mechanism for distributed temperature sensors. Electronics Letters, 1989. 25(14): p. 3.

2. J.M. Lopez-Higuera, E., Hand book of fibre optics sensing technology. 2002: Wiley \& Sons.

3. Boyd, R.W., Non linear Optics. Second ed. 2003, USA: Academic Press; Elsevier Science.

4. Thévenaz, L. Review and progress in distributed fiber sensing. in OFS-18. 2006. Cancún, México: SPIE.

5. Agrawal, G.P., Nonlinear fiber optics. Quantum Electronics Principles and applications, ed. P.F. Liao. 1997, New York: Academic Press.

6. Cho, S.B., J.J. Lee, and I.B. Kwon, Pulse base effect on the strain measurement of a Brillouin-Scattering-based distributed optical fiber sensor. Smart Materials and Structures, 2006. 15: p. 10. 